\title{
Prevalence of Primary Helicobacter pylori Resistance to Several Antimicrobials in a Saudi Teaching Hospital
}

\author{
A.T. Eltahawy \\ Microbiology Laboratory, King Abdulaziz University Hospital, J eddah, Saudi Arabia
}

\section{Key Words}

Helicobacter pylori - Primary resistance - Metronidazole . Clarithromycin · Amoxycillin · Tetracycline.

\begin{abstract}
Objective: To determine the resistance rate of the most commonly used antimicrobial agents amongst Helicobacter pylori isolates. Methods: The agar disk diffusion method (Kirby Bauer) was utilized to determine the susceptibility of $223 \mathrm{H}$. pylori strains isolated before treatment. Isolates were tested against metronidazole $(5 \mu \mathrm{g})$, clarithromycin $(15 \mu \mathrm{g})$, amoxycillin $(10 \mu \mathrm{g})$, and tetracycline $(30 \mu \mathrm{g})$. Results: The resistance rate was $80 \%$ for metronidazole and $4 \%$ for clarithromycin. Tetracycline and amoxycillin showed very low degree of resistance with $1(0.4 \%)$ and $3(1.3 \%)$ of the strains resistant to these antibiotics, respectively. Age, sex and ethnicity had a remarkable effect on the resistance rate. Conclusion: The results indicate that metronidazole and clarithromycin should not be used as the only antimicrobial agents in the treatment of $\mathrm{H}$. pylori infection. Susceptibility testing using the disk diffusion method is cost-effective in the screening of antimicrobial resistance against $\mathrm{H}$. pylori.

\section{Introduction}

Chemotherapy has been documented to be effective in the treatment of Helicobacter pylori and associated gastritis and gastroduodenal ulcers [1-3]. However, this species has not always been successfully eradicated. Unsuccessful therapy in patients infected with $H$. pylori is frequently correlated to metronidazole and clarithromycin resistance [4-6]. Most authorities agree that optimal therapy of $H$. pylori infection requires administration of a minimum of two antibiotics in addition to either a proton pump inhibitor or a hydrogen blocker [7]. Among the many antibiotics examined in vitro, only four are clinically useful: amoxycillin, tetracycline, clarithromycin, and metronidazole. Although resistance to amoxycillin has not yet been described, tetracycline and clarithromycin resistance can be found in up to $16 \%$ of pretreatment isolates [8-10]. Primary metronidazole resistance is more common and more variable, ranging from 10 to $90 \%$ apparently, depending in large part upon the frequency of metronidazole use [8-12]. The correlation between failure to eradicate $H$. pylori with triple antimicrobial therapy (colloidal bismuth subcitrate, metronidazole, amoxycillin or tetracycline) and resistance to metronidazole in pretreatment isolates has been shown worldwide $[6,8,13$, 14]. As a consequence, routine susceptibility testing of H. pylori against metronidazole by standardized methods has been advocated by several investigators [9-15]. The

\footnotetext{
Dr. Ahmed Tarif Abd Elaleem Eltahawy, MBBCh, MSc, PhD (UK)

Consultant Microbiologist

King Abdulaziz University Hospital

PO Box 80215 Jeddah 21589 (Kingdom of Saudi Arabia)

Tel. +9662640 8121, Fax +9662640 3975, E-Mail ahmedeltahawy@hotmail.com
} 
resistance rate of each of the antimicrobials used in the treatment of $H$. pylori infection should be determined in each geographical area and also among different ethnic groups to guide clinical practice. The aim of this study was to determine the resistance pattern of $H$. pylori isolates at King Abdulaziz University Hospital against agents commonly administered for eradication of $H$. pylori infection.

\section{Material and Methods}

\section{Patient Details}

A total of 447 consecutive patients with upper abdominal complaints had endoscopy at the Gastroenterology Unit, King Abdulaziz University Hospital, Jeddah, Saudi Arabia from January 1998 to August 2000. Histology, rapid urease, serology testing and culture were used to determined $H$. pylori status. Patients who had antibiotic treatment of $H$. pylori infection, or bismuth salts less than 1 month prior to endoscopy were excluded from the study. Four biopsy specimens were taken from the antral mucosa during endoscopy; two biopsies were used for histological examination, one for rapid urease (CLO) test, and the other for culture and sensitivity.

\section{Culture}

Biopsy specimens were cultured onto Columbia blood agar, Columbia agar base (Becton Dickinson, Cockeysville, USA) and chocolate agar both supplemented with 5\% defibrinated horse blood (Oxoid, Basingstoke, Hampshire, England). The cultures were incubated in microaerophilic atmosphere (Camp Gas pack, UK) for 5 days. Identification of culture was based on Gram staining, oxidase and catalase activities, and urease positivity. Isolates were stored at $-20^{\circ} \mathrm{C}$ until ready for sensitivity testing.

\section{Antimicrobial Susceptibility}

Susceptibility tests were performed on Mueller-Hinton agar plates (BBL, Cockeysville, USA) supplemented with 5\% horse blood. A standard inoculum of $H$. pylori culture in saline adjusted to 1 McFarland equivalent was used to inoculate the blood agar plates. All isolates were tested for the following antimicrobial agents using Kirby-Bauer disk diffusion (NCCLS) [16]: amoxycillin (10 $\mu \mathrm{g})$, tetracycline $(30 \mu \mathrm{g})$, clarithromycin $(15 \mu \mathrm{g})$, and metronidazole $(5 \mu \mathrm{g})$. Inhibition zone diameters were measured after $48-72 \mathrm{~h}$ of incubation at $37^{\circ} \mathrm{C}$ under microaerophilic conditions.

Reproducibility was checked by testing $H$. pylori type strains NCTC 11637 and H. pylori NCTC 11638. Since there is no precise cutoff point based on the size of the inhibition zone of tested antibiotics, for this study, a zone diameter of $30 \mathrm{~mm}$ or greater was regarded as sensitive, while $29 \mathrm{~mm}$ or less was regarded as resistant.

\section{Results}

Of the 447 biopsies, 223 (50\%) were positive for $H$. pylori, and $214(48 \%)$ were negative. Ten $(2 \%)$ of the biopsies, which had positive cultures of $H$. pylori on pri-
Table 1. Distribution of the zone of inhibition diameters $(\mathrm{mm})$ in relation to the antibiotics tested (number of strains)

\begin{tabular}{lcccc}
\hline \multirow{2}{*}{$\begin{array}{l}\text { Zone } \\
\text { diameter }\end{array}$} & \multicolumn{2}{l}{ Antibiotics } & \\
\cline { 2 - 5 } & metronidazole & clarithromycin & amoxycillin & tetracycline \\
\hline $10-19$ & 170 & 0 & 0 & 0 \\
$20-29$ & 9 & 9 & 3 & 1 \\
$30-39$ & 10 & 13 & 0 & 0 \\
$40-49$ & 7 & 26 & 10 & 6 \\
$50-59$ & 3 & 34 & 23 & 15 \\
$60-69$ & 9 & 39 & 31 & 28 \\
$\geq 70$ & 15 & 102 & 156 & 173 \\
\hline
\end{tabular}

mary isolation, had organisms that died on subculture. Of the 223 isolates tested against the different antimicrobial agents, $179(80.3 \%)$ were resistant to metronidazole agent, $9(4 \%)$ to clarithromycin, $3(1.3 \%)$ to amoxycillin, and 1 strain $(0.4 \%)$ was found resistant to tetracycline. Table 1 shows the distribution of the antibiotic inhibition zone diameters in relation to the different types of antibiotics tested.

The 9 strains resistant to clarithromycin were also resistant to metronidazole. Metronidazole and clarithromycin resistance was more common in females than males. For females, resistance to metronidazole and clarithromycin was 58 and $78 \%$, respectively, while for males the corresponding resistance rate was 42 and $22.2 \%$. There was a remarkable difference in antimicrobial resistance according to age. The distribution of the resistant organisms $(n=192)$ in relation to age-group was as follows: age-group 0-9 years, 0\%; 10-19 years, 2\%; 20-29 years, $14.6 \%$; $30-39$ years, $35 \%$; $40-49$ years, $28 \%$; $50-59$ years, $11.5 \%$; 60-69 years, $7.3 \%$, and age-group $\geq 70$ years, $1.6 \%$. Antibiotic resistance increased gradually up to age 30-39 years, then declined remarkably. Difference in antimicrobial resistance rates in relation to nationality was also observed in this study. The resistance was higher among patients from Somalia, Pakistan and Sudan (6670\%) than among patients from Yemen, Saudi Arabia and Palestine (50-58\%).

\section{Discussion}

Eradication of $H$. pylori species brings an improvement in gastritis and prevents the relapse of gastroduodenal ulcers [7]. The efficacy of treatment of gastric infection caused by $H$. pylori can be reduced by the occurrence 
of primary or acquired resistance to various drugs, especially metronidazole [6]. This has made susceptibility testing of $H$. pylori increasingly important in the search for effective antimicrobial combinations that eradicate these bacteria from the stomach. Test methods used to investigate antimicrobial resistance in $H$. pylori have included agar dilution, microbroth dilution, disk diffusion and E test. However, until now the methods proposed for in vitro susceptibility testing of $H$. pylori have suffered from a lack of standardization and there is still no consensus as to the break points for agents other than clarithromycin used in the treatment of $H$. pylori infection [17]. While both E test and disk diffusion methods have the advantage of allowing the visualization of resistant subpopulations of bacteria within zones of inhibition, disk diffusion testing is less expensive and results in good agreement reference methods [18].

Resistance to metronidazole has been observed worldwide and presently occurs quite commonly in several countries, ranging from 10 to $90 \%$ with the lowest resistance rates in Europe and Australia, the highest rates in Africa whereas in other developing countries, the rate of resistance to metronidazole ranges from 80 to $90 \%$ [12]. The $80 \%$ resistant rate of this study is consistent with that of the previous report [12] and is attributable to previous treatment of gynecologic infection or to the therapy of intestinal parasitic infections, which occur frequently in these countries [19]. However, even when strains were resistant to metronidazole in vitro, a combination of omeperazole, metronidazole, bismuth, and tetracycline given to 165 patients cured $97.6 \%$ of them [20].

The capability of clarithromycin to eradicate $H$. pylori has renewed interest in the macrolides for treating $H$. pylori infection [21] because these compounds achieve high concentrations in mucus and become concentrated intracellularly. Primary resistance to clarithromycin is up to $16 \%[5,9,22,23]$ and is much less common than metronidazole resistance. Based on recent studies, the prevalence of clarithromycin resistance is $10 \%$ in France, $16 \%$ in Italy, $6.8 \%$ in New Zealand and $14.5 \%$ in Iran [8, $9,24,25]$. The low level of resistance (4\%) observed in our study is comparable to these data. However, although the prevalence of resistance to clarithromycin is still low, it may rise with the increased use of this compound for treatment of infections due to both $H$. pylori and other organisms. In contrast to clarithromycin and metronidazole, amoxycillin and tetracycline retained excellent activity. Only 1 strain $(0.4 \%)$ of the $223 \mathrm{H}$. pylori isolates in our study was resistant to tetracycline, and $3(1 \%)$ were resistant to amoxycillin. This high susceptibility of $H$. pylori has also been reported by others [26, 27].

\section{Conclusion}

Susceptibility test using the disk diffusion method is cost-effective in the screening of antimicrobial resistance against $H$. pylori. Testing for resistance to metronidazole and clarithromycin will undoubtedly enable the more rational use of these antibiotics to treat $H$. pylori infection and improve treatment success. It is advisable not to include metronidazole in the treatment regimen in localities where the prevalence of metronidazole resistance is high.

\section{References}

1 Misiewicz JJ, Harris AW, Bardhan KD, Levi S, O'Morain C, Cooper BT, Kerr GD, Dixon MF, Langworthy H, Piper D: One week triple therapy for $H$. pylori: A multicenter comparative study. Gut 1997;41:735-739.

2 Labenz J, Tillenberg B, Weismuller J, Lutke A, Stolte M: Efficacy and tolerability of a one week triple therapy consisting of pantoprazole, clarithromycin and amoxycillin for cure of $H$. pylori infection in patients with duodenal ulcer. Aliment Pharmacol Ther 1997;11:95-100.

3 Lind TF, Megraud F, Bayerdorffer E, O'Morain $\mathrm{C}$, Spiller $\mathrm{R}$, Van Zanten SV, Bardhan $\mathrm{KD}$, Mellblom $\mathrm{H}$, Wrangstadh M, Zeijlon L, Cederberg C: The MACH 2 study: Role of omeperazole in eradication of $H$. pylori with oneweek triple therapies. Gastroenterology 1999; 116:248-253.
4 Bazzoli F, Berretti D, De Luca L, Nicolini G, Pozzato P, Fossi S, Zagan M: What can be learnt from the new data about antibiotic resistance? Are there any practical clinical consequences of $H$. pylori antibiotic resistance? Eur J Gastroenterol Hepatol 1999;11(suppl 2):3942.

5 Osato MS, Reddy R, Graham DY: Metronidazole and clarithromycin resistance amongst $H$. pylori isolates from a large metropolitan hospital in the United States. Int $\mathbf{J}$ Antimicrob Agents 1999;12:341-347.

6 Megraud F: Resistance of $H$. pylori to antibiotics: The main limitation of current protonpump inhibitor triple therapy. Eur J Gastroenterol Hepatol 1999;11(suppl 2):35-37.
7 Nakajima S, Graham DY, Hattori T, Bamba T: Strategy for treatment of $H$. pylori infection in adults: Practical policy in 2000. Curr Pharm Des 2000;6:1515-1529.

8 Fraser AG, Moore L, Hackett M, Hollis B: $H$. pylori treatment and antibiotic susceptibility: Results of a five-year audit. Aust NZ J Med 1999;29:512-516.

9 Street ME, Caruana P, Caffarelli C, Magliani W, Manfredi M, Fornaroli F, de Angelis GL: Antibiotic resistance and antibiotic sensitivity based treatment in H. pylori infection: Advantages and outcome. Arch Dis Child 2001;84: 419-422.

10 Rosandic M: H. pylori resistance in Croatia. Croat Med J 2001;42:45-48. 
11 Realdi G, Dore MP, Piana A, Alzei A, Carta M, Cugia L, Manca A, Are BM, Massarelli G, Mura I, Maida A, Graham DY: Pretreatment antibiotic resistance in $H$. pylori infection: Results of three randomized controlled studies. Helicobacter 1999;4:106-112.

12 Quintana-Guzman EM, Arias-Echandi ML, Salas-Chaves P, Davidovich-Rose H, Schosinsky-Neverman K: H. pylori: Susceptibility to amoxycillin, erythromycin, tetracycline, ciprofloxacin, nitrofurantoin and metronidazole in Costa Rica. Rev Biomed 1998;9:92-96.

13 Midolo PD, Lambert JR, Turnidge J: Metronidazole resistance: A predictor of failure of $H$. pylori eradication by triple therapy. J Gastroenterol Hepatol 1996;3:290-292.

14 Adame KRJ, Suerbaum S, Pfaffenbach B, Opferkuch W: Primary and acquired $H$. pylori resistance to clarithromycin, metronidazole and amoxycillin-influence on treatment outcome. Am J Gastroenterol 1998;93:386-389.

15 Osato MS, Reddy R, Reddy SG, Penland RL, Malaty HM, Graham DY: Pattern of primary resistance of $H$. pylori to metronidazole or chlarithromycin in the United States. Arch Intern Med 2001;161:1217-1220.
16 National Committee for Clinical Laboratory Standards: Performance standard for microbial susceptibility testing (M100-56). Villanova, NCCLS, 1995, vol 15, No 14.

17 Osato MS: Antimicrobial susceptibility testing for $H$. pylori: Sensitivity test results and their clinical relevance. Curr Pharm Des 2000;6: 1545-1555.

18 Midolo PD, Bell JM, Lambert JR, Turnidge JD, Grayson ML: Antimicrobial resistance testing of $H$. pylori: A comparison of E test and disk diffusion methods. Pathology 1997;29: 411-414.

19 Megraud F: Rationale for the choice of antibiotics for the eradication of H. pylori. Eur J Gastroenterol Hepatol 1995; 7(suppl 1):S49-S54.

20 Borody TJ, Andrews P, Francchia G, Brandl S, Shortis NP, Bae H: Omeperazole enhances efficacy of triple therapy in eradicating $H$. pylori. Gut 1995;37:477-481.

21 Rozynek E, Dzierzanowska D, Celinska-Cedro D, Gzyl A, Jelijaszewicz J: Antimicrobial resistance of $H$. pylori in Poland. APUA Newslett 1998;16(2):4-7.
22 Tompkins DS, Perkin J, Smith C: Failed treatment of $H$. pylori infection associated with resistance to clarithromycin. Helicobacter 1997;2:185-187.

23 Occhialini A, Urdaci M, Doucet-Populaire F: Macrolide resistance in $H$. pylori: Rapid detection of point mutations and assays of macrolide binding to ribosomes. Antimicrob Agents Chemother 1997;41:2724-2728.

24 Broutet N, Marais A, Lamouliatte H, de Mascarel A, Samoyeau R, Salamon R, Megraud F: CagA status and eradication treatment outcome of anti-Helicobacter pylori triple therapies in patients with nonulcer dyspepsia. J Clin Microbiol 2001;39:1319-1322.

25 Siavoshi F, Pourkhajeh AH, Merat Sh, AslSoleimani H, Heydriau E, Khatibian M, Malekzadeh R: Susceptibility of various strains of $H$. pylori to selected agent. Arch Iran Med 2000;3:412-415.

26 Graham DY: Antibiotic resistance in $H$. pylori implications for therapy. Gastroenterology 1998;115:1272-1277.

27 Megraud F: Epidemiology and mechanism of antibiotic resistance in $H$. pylori. Gastroenterology 1998;115:1278-1282. 